\title{
CRISPR/dCas9 as a Therapeutic Approach for Neurodevelopmental Disorders: Innovations and Limitations Compared to Traditional Strategies
}

\author{
Raffaele Riccia, ${ }^{a}$ Gaia Colasante ${ }^{a}$ \\ aStem Cell and Neurogenesis Unit, Division of Neuroscience, Ospedale San Raffaele, Milan, Italy; \\ ${ }^{\mathrm{b}}$ Translational and Molecular Medicine PhD Program, DIMET, University of Milan-Bicocca, Milan, Italy
}

\section{Keywords}

Neurodevelopmental disorders · CRISPR/dCas9 - Gene therapy

\begin{abstract}
Brain development is a complex process that requires a series of precise and coordinated events to take place. When alterations in some of those events occur, neurodevelopmental disorders (NDDs) may appear, with their characteristic symptoms, including cognitive, social motor deficits, and epilepsy. While pharmacologic treatments have been the only therapeutic options for many years, more recently the research is turning to the direct removal of the underlying genetic cause of each specific NDD. This is possible thanks to the increased knowledge of genetic basis of those diseases and the enormous advances in genome-editing tools. Together with clustered regularly interspaced short palindromic repeats (CRISPR)/Cas9-based strategies, there is a great development also of nuclease defective Cas9 (dCas9) tools that, with an extreme flexibility, allow the recruitment of specific protein functions to the desired genomic sites. In this work, we review dCas9-based tools and discuss all the published applications in the setting of therapeutic approaches for NDDs at the preclinical level. In particular,
\end{abstract}

dCas9-based therapeutic strategies for Dravet syndrome, transcallosal dysconnectivity caused by mutations in C11orf46 gene, and Fragile $\mathrm{X}$ syndrome are presented and discussed. A direct comparison with other possible therapeutic strategies, such as classic gene replacement or CRISPR/Cas9-based strategies, is provided. We also highlight not only those aspects that constitute a clear advantage compared to previous strategies but also the main technical hurdles related to their applications that need to be overcome.

(c) 2021 S. Karger AG, Base

\section{Introduction}

The correct functionality of the brain is ensured by the accomplishment of complex processes occurring in both embryonic and postnatal life and tightly regulated and coordinated by transcriptional programs [1, 2]. Those processes, which in their entirety constitute the brain development, include proliferation of distinct cell types, differentiation into various fates, migration to their proper locations, and final maturation with integration in local circuits. The final output of these impressive set of events is the brain acquisition of complex abilities like language, cognition, and emotion.
Correspondence to:

Gaia Colasante, colasante.gaia@ hsr.it 
Alterations or complete disruptions of those regulated events can ultimately lead to neurodevelopmental disorders (NDDs) that affect about $3 \%$ of children worldwide $[3,4]$. Typical manifestations of NDDs are impairment in cognition, communication, behavior, and psychomotor abilities and include autism spectrum disorder, intellectual disability (ID), attention-deficit hyperactivity disorder, and epilepsy.

From a genetic perspective, mutations in genes involved in very different developmental pathways are associated with NDDs, including transcriptional and epigenetic regulation, synaptic signaling and neuronal excitability, the signaling of growth factors, and aminoacid and protein synthesis $[5,6]$, evidencing a heterogeneous origin of these disorders. Different types of mutations can occur; not only chromosomal rearrangements, copy number variations, indels, and point mutations but also polygenic origin has been described for some of them [7]. The effect of those mutations may be a "gain of function" (GoF) that is characterized by an increased activity of the mutated gene product not necessarily carrying a dominant-negative effect, or a "loss of function" (LoF) effect, with a reduction of a cellular function.

For a long time, the only therapeutic option for those disorders has been pharmacological treatments; for GoF mutations, they aim to neutralize the mutated protein and/or block its downstream deleterious effects, while in LoF mutations, they try to stimulate the protein function or inhibit the opposite function. In recent years, the effort to define new therapeutic options is shifting toward the reduction of side effects with more specific disease-targeting treatment, that is, precision medicine [8]. This was possible thanks to a deeper understanding of genetic basis of the disease and also to the dramatic advances in DNA manipulation and editing tools.

Classic gene therapy approaches based on gene replacement in cells lacking that specific gene function are the most suitable option for those disorders caused by LoF gene mutations. Nevertheless, it cannot be applied when the coding sequence of the gene exceeds the cargo capacity of currently used viral vectors for gene therapy in the central nervous system (CNS) or when the expression levels of the specific gene need to be carefully dosed as its overexpression may be detrimental $[9,10]$. Furthermore, gene replacement is not an option in the case of GoF mutations, where instead strategies to reduce the expression of mutant target genes, such as antisense oligonucleotides or RNA interference, have been applied $[11,12]$.

The recent advent of clustered regularly interspaced short palindromic repeats (CRISPR)/Cas9 technology
$[13,14]$ has revolutionized functional investigations of gene expression together with the optimization of gene therapy approaches [15]. In comparison to previous gene-editing tools (TALEN and zinc finger proteins), the simplicity of CRISPR/Cas9 tool manipulation has allowed its widespread usage in laboratories with minimal requirement of molecular biology skills and has pushed an enormous development and application of this technique.

Cas9 can be employed to specifically inactivate alleles carrying GoF mutations by insertion of indels at sites where it is recruited by sgRNA to make double-stranded breaks (DSBs; [16-19]) or to directly correct gene mutations in both GoF and LoF mutations upon homology direct recombination (HDR) at DSB sites when a donor DNA is provided [20]. HDR works with high efficiency in dividing cells [21] but not in postmitotic cells like mature neurons. In this case, homology-independent targeted integration has been recently described as a promising option for gene targeting [22], although efficiency is still low to ensure widespread gene correction. Moreover, a major concern for the employment of Cas9-based technologies is that off-target editing may occur $[23,24]$ and may cause permanent DNA alterations with eventual disruption of gene function.

Another possibility in the development of new treatments is in regard to the usage of nuclease defective Cas9 (dCas9), which can become an adaptor to which a variety of effector domains can be attached to address functions in the desired genomic site. In particular, it can be employed to enhance or repress transcription, making it suitable for GoF and LoF mutations, with a reduced risk of off-target compared to Cas9. dCas9 itself can bind target genomic DNA sequences, creating steric hindrance that prevents the activity of other DNA-binding proteins such as endogenous transcription factors and RNA polymerase II and therefore interfering with gene expression (CRISPR interference) [25]. The fusion of dCas9 to a strong repressor complex such as Kruppel-associated Box results in a stronger and more specific gene repression [26]. Similarly, specific induction of gene expression has been achieved by fusing dCas 9 to transcriptional activators in the activatory CRISPR (CRISPRa) [27-30]. dCas9 can also be exploited to recruit various epigenetic writers and erasers to a specific locus. Indeed, dCas 9 has been fused to different epigenetic factors, such as the catalytic domain of eukaryotic DNA methyl transferase 3A (DNMT3A) [31-35] and ten-eleven translocation (TET) proteins to methylate and de-methylate DNA $[36,37]$. dCas9 fusion to histone modifiers, like histone deme- 
Table 1. dCas9-based therapeutic approaches to treat NDDs

\begin{tabular}{|c|c|c|c|c|}
\hline Disease & dCas9 effector domain & Delivery & Outcomes & Ref. \\
\hline \multirow[t]{2}{*}{ Dravet syndrome } & VP160 & $\begin{array}{l}\text { Intracerebroventricular } \\
\text { dual AAV9 injection }\end{array}$ & $\begin{array}{l}\text { Endogenous } \operatorname{Scn} 1 a \text { gene upregulation rescues excitability in } \\
S_{c n} 1 a^{+/-} \text {mutant cortical interneurons in vitro and improves febrile } \\
\text { seizures phenotype in vivo in } S c n 1 a^{+/-} \text {mice }\end{array}$ & {$[42]$} \\
\hline & VP64-p65-Rta (VPR) & $\begin{array}{l}\text { Intravenous PHP.eB } \\
\text { injection }\end{array}$ & $\begin{array}{l}\text { Endogenous } S c n 1 a \text { gene upregulation ameliorates febrile seizures } \\
\text { and improves behavioral alterations in floxed-dCas9-VPR } \\
\text { Vgat-Cre/Scn } 1 a^{\mathrm{RX} /+} \text { mice }\end{array}$ & {$[43]$} \\
\hline $\begin{array}{l}\text { Transcallosal } \\
\text { dysconnectivity }\end{array}$ & SunTag-C11orf46 & In utero electroporation & $\begin{array}{l}\text { Repression of Sema6a gene expression rescues impaired midline } \\
\text { crossing of callosal projections and axonal arborization deficit in } \\
\text { vivo }\end{array}$ & {$[59]$} \\
\hline Fragile X syndrome & TET1 & $\begin{array}{l}\text { Lentiviral transduction } \\
\text { in vitro }\end{array}$ & $\begin{array}{l}\text { Demethylation of CGG expansion in FMR1 promoter region } \\
\text { restores FMR1 expression in fragile X syndrome patient derived } \\
\text { iPSCs and induced neurons }\end{array}$ & {$[84]$} \\
\hline
\end{tabular}

thylase LSD1, that removes the active enhancer marker H3K4me2 mark [38], and histone acetyl transferase P300 [39] result in the reduction and increase of enhancer activity, respectively. dCas9-SunTag is a scaffold protein made of a repeated peptide array, which can recruit multiple copies (up to 24) of a desired regulatory protein to a genomic site, thanks to the fusion of the specific effector protein to an antibody with high affinity to that peptide array [40]. Finally, dCas9 fusion to an engineered reverse transcriptase makes it possible to rewrite new genetic information into a specified DNA site; in this case, the prime editing exploits a guide RNA (prime editing guide RNA) that both addresses the dCas9 to the specific target genomic region and encodes the edit to be introduced at the same time [41].

All these tools represent a gold mine in which many laboratories have dived to make the most of them. In the present work, we aimed to review all the therapeutic approaches selectively targeting NDDs based on CRISPR/ dCas9 that are being developed at the preclinical level (Table 1), highlighting those aspects that could give them an advantage compared to previous strategies and main limitations.

\section{CRISPR/dCas9-Based Applications in Models of NDDs}

CRISPRa has been recently exploited to recover the haploinsufficiency of Scn 1a gene [42, 43], a gene encoding for the alpha subunit of voltage-gated sodium channel $\mathrm{Na}_{\mathrm{v}}$ 1.1. LoF mutations in SCN1A determine a spectrum of disorders with different phenotypic severity, ranging from febrile seizures plus to generalized epilepsy with febrile seizures plus and Dravet syndrome (DS), a severe infantile epilepsy that begins during the first year of life and leads to severe motor, cognitive, and social interaction deficits [44]. A strong contribution of the genetic background on the phenotypic manifestations of the same mutation has been reported $[45,46] . \mathrm{Na}_{\mathrm{v}} 1.1$ is enriched at the axonal initial segmentof GABAergic interneurons - although more and more evidences regarding its expression in glutamatergic neurons are emerging [47, 48] - where it plays a critical role in AP generation. As a consequence, Scn 1a LoF mutations imply decreased excitability of inhibitory neurons, which appears to be the underlying cause of intractable epilepsy [49]. Different animal models of DS that well recapitulate both the epileptic phenotype and behavioral alterations are available $[50,51]$.

DS is the ideal candidate for the application of a gene therapy based on CRISPRa either because the underlying genetic mechanism is gene haploinsufficiency or because the coding sequence of $S \mathrm{cn}$ la gene, which is longer than $6 \mathrm{~kb}$, cannot be accommodated in adeno-associated viral vectors (AAVs), commonly employed for gene delivery in the CNS. With these premises, increasing the expression of the healthy copy of Scnla looks like a promising approach, and other strategies based on the same rationale have been developed $[52,53]$.

The two studies $[42,43]$ start with a screening of sgRNAs targeting $S c n 1 a$ distal and proximal promoters in a cell line in vitro. Several sgRNAs were tested for their ability to increase basal levels of Scn 1a in those cells in association with dCas9 fused to different transcriptional activators, specifically VP160 [42] and VP64-p65-Rta (VPR) [43]. Surprisingly, they came to different conclusions, as one laboratory defined efficacious sgRNAs tar- 
geting the distal promoter [43], while the other a unique upregulating sgRNA targeting Scnla proximal promoter [42]. Interestingly, this latter Scn1a promoter (1b) has been recently described as a critical disease-relevant regulatory element. In fact, its ablation in a mouse model is sufficient to induce Dravet phenotype [54], suggesting that this region, before considered as a redundant promoter, can have instead a pivotal regulatory function for Scnla gene.

Colasante and colleagues [42] performed a complete characterization of the effect of CRISPRa in primary neurons in vitro before moving to test its effect in vivo, showing that the selected sgRNA was able to upregulate specifically Scn1a gene without any relevant effect on predicted off-target genes. The Scn 1a-dCas9A treatment can increase $\mathrm{Na}_{\mathrm{v}} 1.1$ protein level in Dravet primary neurons, and it is sufficient to induce a recovery of Dravet GAD67 + GABAergic interneuron firing rates [42].

Both studies also provide a proof of principle of their efficacy in vivo. The first study proposes an approach based on the intracerebroventricular delivery in perinatal Dravet mice pups of Scn1a-dCas9A tool by a dual AAV system (as also activatory dCas9 is a large protein) [42]. Yamagata and colleagues [43] crossed Dravet mice with dCas9VPR mice, while sgRNAs were delivered by systemic injection of PhPeB.AAV [55] in juvenile mice at 4 weeks of age.

Interestingly, taking into account that $\mathrm{Na}_{\mathrm{v}} 1.1$ is expressed also in pyramidal neurons where its overexpression may be detrimental [47], both strategies restricted Scn1a gene upregulation to GABAergic inhibitory interneurons either by the choice of $\mathrm{mDlx} 5 / 6$ enhancer [56] to drive the dCas9 expression cassette [42] or by using a further genetic crossing to express dCas9VPR only in vesicular GABA aminoacid transporter neurons [43].

In both studies, Scn1a-dCas9A treatment of Scn1a+/mice could ameliorate febrile seizures, increasing the temperature threshold for seizure induction $[42,43]$. Yamagata and colleagues [43] expanded their in vivo analysis, showing a modest effect on non-epileptic alterations.

Ultimately, both studies, each with their specific limitations - delivery in pups or poor phenotypic amelioration - do not answer the open question of symptomatic reversibility in DS. In fact, in one study Scn1a-dCas9A is conceived as a preventive treatment (perinatally in pups), while in the other it is delivered in 4-week-old mice, but poor effect on phenotypic manifestations was observed. Therefore, they leave still open the possibility that increasing Scnla gene is not efficacious in treating the pathology in the chronic phase of the disease [57], when normalization of interneuron activity occurs [58] but seizures are still present.

An evolution of dCas9-SunTag [40] has been recently exploited to set a treatment in transcallosal dysconnectivity caused by mutations in C11orf46 gene [59]. As described above, the main advantage of the dCas9-SunTag is its ability to recruit multiple copies of the protein of interest onto a single sgRNA-target sequence, providing a stronger effect in comparison to " $1: 1$ " systems (one transcriptional regulator for each dCas9 molecule).

Disrupted interhemispheric communication due to defects in the corpus callosum is the cause of some NDDs manifesting with ID, autism spectrum disorder, and schizophrenia [60-62]. Point mutation genes encoding for signaling molecules encoding regulators of neurite outgrowth and axon guidance, such as the Roundabout guidance receptor 1 (ROBO1) and the $L 1$ cell adhesion molecule (L1CAM), have already been described as the cause of callosal connectivity $[63,64]$. Peter and colleagues [59] first reported that the haploinsufficiency of the small nuclear protein C11orf46/ADP ribosylation factor like GTPase 14 effector protein (ARL14EP), encoded in the chr. 11p13 Wilms tumor, aniridia, genitourinary abnormalities, and ID risk locus, causes callosal hypoplasia in patients. In rodents, C11orf46 is primarily expressed in postmitotic and post-migratory glutamatergic cortical neurons, and its silencing in mouse embryonic brain development impairs the projection formation in cortical neurons and transcallosal connectivity [59]. Interestingly, they identified C11orf46 as a key binding partner of the repressor complex SET domain bifurcated histone lysine methyltransferase 1 (SETDB1)/KMT1E (SETDB2/ KMT1F) MBD-containing-associated factor histone lysine 9 (K9) methyltransferase (KMT-RC). Accordingly, RNA-seq data showed that the expression of some genes relevant for axon guidance, like Semaphorin $6 a$ (Sema6a), was increased upon C11orf46 knockdown, supporting the pivotal role of this protein in the repressor complex KMT-RC [59]. Given this, C11orf46's affinity to the KMT-RC complex was then exploited to alter neuronal gene expression via a chromatin-associated mechanism. The dCas9-SunTag protein scaffold system was chosen to load 10 copies of C11orf46 and recruit KMT-RC on Sema6a gene locus and repress its expression. The modular dCas9-SunTag has been previously shown to be able to recruit multiple DNMT3A catalytic domains to a target site for editing DNA methylation in a way that is more tunable, specific (strong decrease of off-target methylation), and more efficient in providing DNA methylation at target sites than the direct fusion of dCas9 to DNMT3A 
[65]. Strikingly, this dCas9-SunTag-C11 orf46 targeting the Sema6a promoter, when expressed during mouse brain development, rescued impaired midline crossing of callosal projections and axonal arborization deficit normally encountered upon C11orf46 knockdown [59].

While this study has the value to pose the basis for recruiting any protein function in a specific locus and setting future chromatin-based therapies to correct developmental alterations in the brain's connectome, the direct advantages of using an epigenomic approach instead of a classic gene therapy based on the re-expression of C11orf46 is not clearly highlighted. In fact, gene replacement of C11 orf 46 would have been able to restore the normal expression for all the C11orf46/KMT-RC direct target genes, although probably with less efficiency.

More straightforward is the application of a dCas9based epigenetic approach to treat the fragile X chromosome syndrome (FXS). FXS is an X-linked NDD and produces a complex disorder with a range of neurological and psychiatric problems, including in most cases autistic features with intellectual, cognitive, and social alterations [66-69]. The underlying cause is the loss of expression of the fragile $\mathrm{X}$ mental retardation protein (FMRP) encoded by the FMR1 gene during neurodevelopment [70-72]. FMR1 gene silencing is caused by hypermethylation of its promoter [73], due to a CGG trinucleotide repeat expansion mutation at the $5^{\prime}$ UTR of FMR1, which in healthy individuals is made approximately of 6-44 repeats, whereas FXS patients show more than 200 repeats [74, 75]. FMRP is an RNA-binding protein in neurons that controls protein synthesis at developing synapses and has a key role in the maintenance of synaptic plasticity $[68,76,77]$.

Interestingly, when knock-in mice carrying different lengths of CGG repeat expansions (even >300) were generated, no increased methylation of the Fmrl gene was found, and these mice displayed increased mRNA level but decreased Fmrp protein with a phenotype that is accordingly more similar to fragile $\mathrm{X}$ premutation carriers (with expansion <200) than to FXS patients [78]. Therefore, the epigenetic silencing seems to be a human-specific mechanism.

The lack of an adequate mouse model accelerated the generation of human cellular models such as embryonic stem cells and induced pluripotent stem cells (iPSCs) derived from patients. Loss of FMRP expression in iPSCderived neurons leads to deregulated production and membrane insertion of neurotransmitter receptors and ion channels, causing synaptic hyper-excitability that affects proper function of various neural circuits in the CNS $[67,69,79]$.

CRISPR/dCas9-Based Therapies in

Neurodevelopmental Disorders
The first attempts of epigenetic editing were done with non-specific demethylating agents [80-83], but these drugs are usually too toxic to be employed as a therapy in FXS patients, as they are not specific at all and demethylation is a universal method of regulating gene expression.

Recently, Dr. Rudolf Jaenisch lab demonstrated that the recruitment of dCas9-TET fusions to CGG repeats caused robust loss of methylation at the FMR1 promoter in human cells, associated with almost complete restoration of FMRP protein expression [84]. Demethylation of the CGG expansion increased histone H3 lysine 27 (H3K27) acetylation and H3K4 trimethylation, and decreased $\mathrm{H} 3 \mathrm{~K} 9$ trimethylation at the FMR1 promoter region, which finally induced FMR1 gene reactivation. RNA sequencing and whole-genome bisulfite sequencing (to measure DNA methylation changes) demonstrated that transcriptional and epigenetic effects were highly specific to the targeted FMR1 locus. Interestingly, FMR1 expression and demethylation of its promoter were maintained, at least for 2 weeks, in the presence of AcrIIA4, an inhibitor of Cas9/dCas9 [85]. Epigenetic editing was maintained also in iPSC-derived neurons in which the increased firing rate was rescued [84]. Also, neurons transplanted in mouse brains maintained FMR1 reactivation up to 3 months [84].

When the demethylation of the FMR1 promoter was performed directly in iPSC-derived neurons, the reactivation of the FMR1 gene was less robust than that seen in iPSCs [84], probably because DNA demethylation mechanisms are less efficient in postmitotic cells $[86,87]$. Despite the lack of a murine model to directly test the dCas9TET efficacy on demethylating FMR1 locus in vivo, this analysis on the stability of demethylation on transplanted neurons is promising for a long-term effect of the treatment. Finally, in comparison to the classic CRISPR/Cas9editing system that had been successfully exploited to eliminate or shorten the CGG repeats in hiPSCs carrying FXS expansion $[88,89]$, the epigenetic strategy has some advantages that will be highlighted in the next section.

\section{CRISPR/dCas9 Approaches: Advantages and}

\section{Limitations}

The extensive usage of CRISPR/dCas9 tools attests their attractivity and their potential for the treatment of NDDs. Indeed, they present some indisputable advantages in comparison to previously available therapeutic strategies.

First, in comparison to classic gene replacement, CRISPRa/dCas9 tools can boost gene expression, acting directly on endogenous genes, without any limitation of

Dev Neurosci 2021;43:253-261 
gene size. In addition, as they allow the expression of the complete mRNA of the target gene, its physiological splicing dynamics remain unaltered. Also, the $3^{\prime} U T R$ of the target genes, which has a well-established role in the nuclear export, subcellular targeting, and rates of translation and degradation of mRNA $[90,91]$, is maintained. This aspect is particularly relevant when significant alterations of gene dosage may be detrimental, such as for Mecp2 gene [92, 93].

In comparison to nucleasic CRISPR/Cas9, the dCas9 has the advantage of being able to modulate gene expression by non-permanent changes in the genome, making them more suitable in a translational perspective. Moreover, the effect of eventual off-target genes is potentially less dangerous with respect to DSBs that might occur at Cas9 off-target sites. This is particularly meaningful in light of recent evidence indicating that AAVs - carrying, for example, Cas 9 or donors for HDR - can integrate with a relatively high frequency into Cas9-generated DSB sites [94-96].

On the other hand, nucleasic Cas9-based systems induce permanent modification in the genome, implying that its restricted expression in a short time window is sufficient to induce the desired editing. Conversely, CRIS$\mathrm{PRa} / \mathrm{dCas} 9$ tools rely on the continuous expression of dCas9 in the cells, increasing the risk of neuronal toxicity [43] and of the potential immunogenic response to the Cas9 bacterial protein [97]. Epigenetic changes induced by dCas 9 seem to be maintained also in the presence of Cas9 inhibitor [84], suggesting that constitutive presence of dCas9 may not be required. However, most of the studies lack a long-term effect of CRISPR/dCas9 epigenetic hit and the real maintenance of the epigenetic mark should be tested in each single case.

A major technical hurdle related to in vivo application of CRISPR/dCas9-based tools for the treatment of NDDs is the delivery in the CNS. In fact, packaging in AAVs Streptococcus pyogenes SpdCas9 with its extra fused protein functions together with the sgRNA cassette is a real challenge, and there is no room to introduce any additional regulatory elements, such as neuronal subtype-specific promoters. The identification of the shorter ortholog derived from Staphylococcus aureus (SaCas9, 1,053 amino acids) [98] has only partially solved the AAV cargo issue. For this reason, many in vivo applications are still based on dual AAV systems, in which each of the two AAVs carries some of the elements that need to be delivered [42] or carries a "split dCas9" system based on an intein protein that can mediate dCas9 trans-splicing [99, 100]. In both cases, cell co-transduction with the two
AAVs is needed to achieve the CRISPR/dCas9 therapeutic effect, a requirement that decreases the efficiency of the treatment.

In conclusion, while numerous hurdles still exist for the delivery of CRISPR tools to the CNS, there are more and more preclinical studies for NDDs that offer the hope that transcriptional CRISPR/dCas9 modulation or epigenetic editing could be used in human diseases that affect brain function in the next future. In the process of development of those therapies, the acquisition of more knowledge regarding the basic biology of the pathologies would be of help. Understanding if a specific NDD can be reverted after symptom onset, defining the ideal therapeutic window, and identifying the minimal region of the brain or the minimal number of cells/neuronal subtype to be corrected to achieve a significant phenotypic amelioration would produce an acceleration of therapeutic translation of those approaches.

\section{Conflict of Interest Statement}

The authors have no conflicts of interest to declare.

\section{Funding Sources}

This work was supported by the "Associazione Gruppo Famiglie Dravet" to G.C.; CARIPLO Foundation (2016-0532) and the Italian Ministry of Health (GR-2016-02363972) to G.C.; and Telethon GGP19249 to G.C.

\section{Author Contributions}

R.R. and G.C. wrote the manuscript.

References

1 Silbereis JC, Pochareddy S, Zhu Y, Li M, Ses$\tan \mathrm{N}$. The cellular and molecular landscapes of the developing human central nervous system. Physiol Behav. 2017;176(1):139-48.

2 Yap EL, Greenberg ME. Activity-regulated transcription: bridging the gap between neural activity and behavior. Neuron. 2018; 100(2):330-48.

3 Gilissen C, Hehir-Kwa JY, Thung DT, Van De Vorst M, Van Bon BW, Willemsen MH, et al. Genome sequencing identifies major causes of severe intellectual disability. Nature. 2014; 511(7509):344-7.

4 Ismail FY, Shapiro BK. What are neurodevelopmental disorders?. Curr Opin Neurol. 2019;32(4):611-6. 
5 Parenti I, Rabaneda LG, Schoen H, Novarino G. Neurodevelopmental disorders: from genetics to functional pathways. Trends Neurosci. 2020;43(8):608-21.

6 Turner TJ, Zourray C, Schorge S, Lignani G. Recent advances in gene therapy for neurodevelopmental disorders with epilepsy. J Neurochem. 2020(June):1-34.

$7 \mathrm{Hu}$ WF, Chahrour MH, Walsh CA. The diverse genetic landscape of neurodevelopmental disorders. Annu Rev Genomics Hum Genet. 2014;15:195-213.

8 Ashley EA. Towards precision medicine. Nat Rev Genet. 2016;17(9):507-22.

9 Meins M, Lehmann J, Gerresheim F, Herchenbach J, Hagedorn M, Hameister K, et al. Submicroscopic duplication in Xq28 causes increased expression of the MECP2 gene in a boy with severe mental retardation and features of Rett syndrome. J Med Genet. 2005; 42(2):e12-6.

10 Oostra BA, Willemsen R. A fragile balance: FMR1 expression levels. Hum Mol Genet. 2003;12 Spec No 2(REV. ISS. 2):R249-57.

11 Rinaldi C, Wood MJA. Antisense oligonucleotides: the next frontier for treatment of neurological disorders. Nat Rev Neurol. 2018; 14(1):9-21.

12 Setten RL, Rossi JJ, Han SP. The current state and future directions of RNAi-based therapeutics. Nat Rev Drug Discov. 2019;18(6): $421-46$.

13 Knott GJ, Doudna JA. CRISPR-Cas guides the future of genetic engineering. Science. 361(6405):866-9.

14 Zhang F. Development of CRISPR-Cas systems for genome editing and beyond. Quart Rev Biophys. 2019;52.

15 Doudna JA. The promise and challenge of therapeutic genome editing. Nature. 2020; 578(7794):229-36.

16 Dai WJ, Zhu LY, Yan ZY, Xu Y, Wang QL, Lu XJ. CRISPR-Cas9 for in vivo gene therapy: promise and hurdles. Mol Ther Nucleic Acids. 2016;5:e349.

17 Wang D, Zhang F, Gao G. CRISPR-based therapeutic genome editing: strategies and in vivo delivery by AAV vectors. Cell. 2020; 181(1):136-50.

18 Christie KA, Robertson LJ, Conway C, Blighe $\mathrm{K}$, DeDionisio LA, Chao-Shern C, et al. Mutation-independent allele-specific editing by CRISPR-Cas9, a novel approach to treat autosomal dominant disease. Mol Ther. 2020 28(8):1846-57.

19 Gao X, Tao Y, Lamas V, Huang M, Yeh WH Pan B, et al. Treatment of autosomal dominant hearing loss by in vivo delivery of genome editing agents. Nature. 2018;553(7687): 217-21.

20 Pawelczak KS, Gavande NS, VanderVereCarozza PS, Turchi JJ. Modulating DNA repair pathways to improve precision genome engineering. ACS Chem Biol. 2018;13(2): 389-96.

21 Lin S, Staahl BT, Alla RK, Doudna JA. Enhanced homology-directed human genome engineering by controlled timing of CRISPR/ Cas9 delivery. Elife. 2014;3:e04766.

22 Suzuki K, Tsunekawa Y, Hernandez-Benitez $\mathrm{R}, \mathrm{Wu}$ J, Zhu J, Kim EJ, et al. In vivo genome editing via CRISPR/Cas9 mediated homology-independent targeted integration. Nature. 2016;540(7631):144-9.

$23 \mathrm{Fu} \mathrm{Y}$, Foden JA, Khayter C, Maeder ML, Reyon D, Joung JK, et al. High-frequency offtarget mutagenesis induced by CRISPR-Cas nucleases in human cells. Nat Biotechnol. 2013;31(9):822-6.

24 Pattanayak V, Lin S, Guilinger JP, Ma E, Doudna JA, Liu DR. High-throughput profiling of off-target DNA cleavage reveals RNAprogrammed Cas 9 nuclease specificity. Nat Biotechnol. 2013;31(9):839-43.

25 Qi LS, Larson MH, Gilbert LA, Doudna JA, Weissman JS, Arkin AP, et al. Repurposing CRISPR as an RNA-guided platform for sequence-specific control of gene expression. Cell. 2013;152(5):1173-83.

26 Thakore PI, D'Ippolito AM, Song L, Safi A, Shivakumar NK, Kabadi AM, et al. Highly specific epigenome editing by CRISPR-Cas9 repressors for silencing of distal regulatory elements. Nat Methods. 2015;12(12):1143-9.

27 Mali P, Esvelt KM, Church GM. Cas9 as a versatile tool for engineering biology. Nat Methods. 2013;10(10):957-63.

28 Perez-Pinera P, Kocak DD, Vockley CM, Adler AF, Kabadi AM, Polstein LR, et al. RNA-guided gene activation by CRISPR Cas9-based transcription factors. Nat Meth ods. 2013;10(10):973-6.

29 Chavez A, Scheiman J, Vora S, Pruitt BW, Tuttle M, P R Iyer EE, et al. Highly efficient Cas9-mediated transcriptional programming. Nat Methods. 2015;12(4):326-8.

30 Konermann S, Brigham MD, Trevino AE, Joung J, Abudayyeh OO, Barcena C, et al. Genome-scale transcriptional activation by an engineered CRISPR-Cas9 complex. Nature. 2015;517(7536):583-8.

31 Amabile A, Migliara A, Capasso P, Biffi M, Cittaro D, Naldini L, et al. Inheritable silencing of endogenous genes by Hit-and-Run targeted epigenetic editing. Cell. 2016;167(1): 219-e14.

32 McDonald JI, Celik H, Rois LE, Fishberger G, Fowler T, Rees R, et al. Reprogrammable CRISPR/Cas9-based system for inducing sitespecific DNA methylation. Biol Open. 2016; 5(6):866-74.

33 Vojta A, Dobrinić P, Tadić V, Bočkor L, Korać $\mathrm{P}$, Julg B, et al. Repurposing the CRISPR-Cas9 system for targeted DNA methylation. Nucleic Acids Res. 2016;44(12):5615-28.

34 Xiong T, Meister GE, Workman RE, Kato NC, Spellberg MJ, Turker F, et al. Targeted DNA methylation in human cells using engineered dCas9-methyltransferases. Sci Rep. 2017;7(1): 6732-14.

35 Liu XS, Wu H, Ji X, Stelzer Y, Wu X, Czauderna $S$, et al. Editing DNA methylation in the mammalian genome. Cell. 2016;167(1):233e17.
36 Morita S, Noguchi H, Horii T, Nakabayashi K, Kimura M, Okamura K, et al. Targeted DNA demethylation in vivo using dCas9peptide repeat and scFv-TET1 catalytic domain fusions. Nat Biotechnol. 2016;34(10): 1060-5.

37 Xu X, Tao Y, Gao X, Zhang L, Li X, Zou W, et al. A CRISPR-based approach for targeted DNA demethylation. Cell Discov. 2016;2: 16009.

38 Shi Y, Lan F, Matson C, Mulligan P, Whetstine JR, Cole PA, et al. Histone demethylation mediated by the nuclear amine oxidase homolog LSD1. Cell. 2004;119(7):941-53.

39 Hilton IB, D'Ippolito AM, Vockley CM, Thakore PI, Crawford GE, Reddy TE, et al. Epigenome editing by a CRISPR-Cas9-based acetyltransferase activates genes from promoters and enhancers. Nat Biotechnol. 2015; 33(5):510-7.

40 Tanenbaum ME, Gilbert LA, Qi LS, Weissman JS, Vale RD. A protein-tagging system for signal amplification in gene expression and fluorescence imaging. Cell. 2014;159(3): 635-46.

41 Anzalone AV, Randolph PB, Davis JR, Sousa AA, Koblan LW, Levy JM, et al. Search-andreplace genome editing without doublestrand breaks or donor DNA. Nature. 2019; 576(7785):149-57.

42 Colasante G, Lignani G, Brusco S, Di Berardino C, Carpenter J, Giannelli S, et al. dCas9based Scnla gene activation restores inhibitory interneuron excitability and attenuates seizures in Dravet syndrome mice. Mol Ther. 2020;28(1):235-53.

43 Yamagata T, Raveau M, Kobayashi K, Miyamoto $\mathrm{H}$, Tatsukawa T, Ogiwara I, et al. CRISPR/ dCas9-based Scn1a gene activation in inhibitory neurons ameliorates epileptic and behavioral phenotypes of Dravet syndrome model mice. Neurobiol Dis. 2020;141(May):104954.

44 Catterall WA, Kalume F, Oakley JC. Nav1.1 channels and epilepsy. J Physiol. 2010; 588(11):1849-59.

45 Marini C, Scheffer IE, Nabbout R, Mei D, Cox K, Dibbens LM, et al. SCN1A duplications and deletions detected in Dravet syndrome: implications for molecular diagnosis. Epilepsia. 2009;50(7):1670-8.

46 Cetica V, Chiari S, Mei D, Parrini E, Grisotto L, Marini C, et al. Clinical and genetic factors predicting Dravet syndrome in infants with SCN1A mutations. Neurology. 2017;88(11): 1037-44.

47 Ogiwara I, Iwasato T, Miyamoto H, Iwata R, Yamagata T, Mazaki E, et al. Nav1.1 haploinsufficiency in excitatory neurons ameliorates seizure-associated sudden death in a mouse model of dravet syndrome. Hum Mol Genet. 2013;22(23):4784-804.

48 Almog Y, Fadila S, Brusel M, Mavashov A, Anderson K, Rubinstein M. Developmental alterations in firing properties of hippocampal CA1 inhibitory and excitatory neurons in a mouse model of Dravet syndrome. Neurobiol Dis. 2021;148:105209.
CRISPR/dCas9-Based Therapies in Neurodevelopmental Disorders
Dev Neurosci 2021:43·253-261 
49 Yu FH, Mantegazza M, Westenbroek RE, Robbins CA, Kalume F, Burton KA, et al. Reduced sodium current in GABAergic interneurons in a mouse model of severe myoclonic epilepsy in infancy. Nat Neurosci. 2006; 9(9):1142-9.

50 Griffin A, Hamling KR, Hong S, Anvar M, Lee LP, Baraban SC. Preclinical animal models for Dravet syndrome: seizure phenotypes, comorbidities and drug screening. Front Pharmacol. 2018;9(Jun):573.

51 Ricobaraza A, Mora-Jimenez L, Puerta E, Sanchez-Carpintero R, Mingorance A, Artieda J, et al. Epilepsy and neuropsychiatric comorbidities in mice carrying a recurrent Dravet syndrome SCN1A missense mutation. Sci Rep. 2019;9(1):14172-15.

52 Hsiao J, Yuan TY, Tsai MS, Lu CY, Lin YC, Lee ML, et al. Upregulation of haploinsufficient gene expression in the brain by targeting a long non-coding RNA improves seizure phenotype in a model of Dravet syndrome. EBioMedicine. 2016;9:257-77.

53 Han Z, Chen C, Christiansen A, Ji S, Lin Q, Anumonwo C, et al. Antisense oligonucleotides increase Scnla expression and reduce seizures and SUDEP incidence in a mouse model of Dravet syndrome. Sci Transl Med. 2020;12(558):eaaz6100.

54 Haigh JL, Adhikari A, Copping NA, Stradleigh T, Wade AA, Catta Preta R et al. Deletion of a non-canonical regulatory sequence causes loss of Scn1a expression and epileptic phenotypes in mice. bioRxiv. 2021.

55 Chan KY, Jang MJ, Yoo BB, Greenbaum A, Ravi N, Wu WL, et al. Engineered AAVs for efficient noninvasive gene delivery to the central and peripheral nervous systems. Nat Neurosci. 2017;20(8):1172-9.,

56 Dimidschstein J, Chen Q, Tremblay R, Rogers SL, Saldi GA, Guo L, et al. A viral strategy for targeting and manipulating interneurons across vertebrate species. Nat Neurosci. 2016; 19(12):1743-9.

57 Goldberg EM. Gene therapy in models of severe epilepsy due to sodium channelopathy. Epilepsy Curr. 2020;20(4):214-7.

58 Favero M, Sotuyo NP, Lopez E, Kearney JA, Goldberg EM. A transient developmental window of fast-spiking interneuron dysfunction in a mouse model of dravet syndrome. Neurosci. 2018;38(36):7912-27.

59 Peter CJ, Saito A, Hasegawa Y, Tanaka Y, Nagpal M, Perez G, et al. In vivo epigenetic editing of Sema6a promoter reverses transcallosal dysconnectivity caused by C11orf46/Arl14ep risk gene. Nat Commun. 2019;10(1):4112.

60 Peiker I, David N, Schneider TR, Nolte G, Schöttle D, Engel AK. Perceptual integration deficits in autism spectrum disorders are associated with reduced interhemispheric gamma-band coherence. J Neurosci. 2015;35(50): 16352-61.

61 Ribolsi M, Daskalakis ZJ, Siracusano A, Koch G. Abnormal asymmetry of brain connectivity in schizophrenia. Front Hum Neurosci. 2014;8(Dec):1010-1.
62 Margari L, Palumbi R, Campa MG, Operto FF, Buttiglione M, Craig F, et al. Clinical manifestations in children and adolescents with corpus callosum abnormalities. J Neurol. 2016;263(10):1939-45.

63 Calloni SF, Cohen JS, Meoded A, Juusola J, Triulzi FM, Huisman TAGM, et al. Compound heterozygous variants in $\mathrm{ROBO} 1$ cause a neurodevelopmental disorder with absence of transverse pontine fibers and thinning of the anterior commissure and corpus callosum. Pediatr Neurol. 2017;70:70-4.

64 Shieh C, Moser F, Graham JM, Watiker V, Pierson TM. Mutation in the sixth immunoglobulin domain of L1CAM is associated with migrational brain anomalies. Neurol Genet. 2015;1(4):e34-6.

65 Pflueger C, Tan D, Swain T, Nguyen T, Pflueger J, Nefzger C, et al. A modular dCas9SunTag DNMT3A epigenome editing system overcomes pervasive off-target activity of direct fusion dCas9-DNMT3A constructs. Genome Res. 2018;28(8):1193-206.

66 Pugin A, Faundes V, Santa María L, Curotto B, Aliaga S, Salas I, et al. Aspectos clínicos, moleculares y farmacológicos en los trastornos asociados a gen 1 del retraso mental del $\mathrm{X}$ frágil. Neurología. 2017;32(4):241-52.

67 Santoro MR, Bray SM, Warren ST. Molecular mechanisms of fragile X syndrome: a twentyyear perspective. Annu Rev Pathol. 2012;7: 219-45.

68 Sidorov MS, Auerbach BD, Bear MF. Fragile $\mathrm{X}$ mental retardation protein and synaptic plasticity. Mol Brain. 2013;6(1):15-1.

69 Contractor A, Klyachko VA, Portera-Cailliau C. Altered neuronal and circuit excitability in Fragile X syndrome. Neuron. 2015;87(4): 699-715.

70 Crawford DC, Acuña JM, Sherman SL, Med G. FMR1 and the Fragile X syndrome: human genome epidemiology review HHS public access author manuscript. Genet Med. 2001; 3(5):359-71. Available from: https://europepmc.org/backend/ptpmcrender. fcgi?accid=PMC4493892\&blobtype $=$ pdf.

71 O'Donnell WT, Warren ST. A decade of molecular studies of fragile X syndrome. Annu Rev Neurosci. 2002;25:315-38.

72 Penagarikano O, Mulle JG, Warren ST. The pathophysiology of fragile X syndrome. Annu Rev Genomics Hum Genet. 2007;8:109-29.

73 Kumari D, Gazy I, Usdin K. Pharmacological reactivation of the silenced FMR1 gene as a targeted therapeutic approach for fragile $\mathrm{X}$ syndrome. Brain Sci. 2019;9(2):39.

74 Fu YH, Kuhl DP, Pizzuti A, Pieretti M, Sutcliffe JS, Richards S, et al. Variation of the CGG repeat at the fragile $\mathrm{X}$ site results in genetic instability: resolution of the Sherman paradox. Cell. 1991;67(6):1047-58.

75 Verkerk AJ, Pieretti M, Sutcliffe JS, Fu YH, Kuhl DP, Pizzuti A, et al. Identification of a gene (FMR-1) containing a CGG repeat coincident with a breakpoint cluster region exhibiting length variation in fragile $\mathrm{X}$ syndrome. Cell. 1991;65(5):905-14.
76 Darnell JC, Van Driesche SJ, Zhang C, Hung KY, Mele A, Fraser CE, et al. FMRP stalls ribosomal translocation on mRNAs linked to synaptic function and autism. Cell. 2011; 146(2):247-61.

77 Smith LN, Jedynak JP, Fontenot MR, Hale CF, Dietz KC, Taniguchi M, et al. Fragile X mental retardation protein regulates synaptic and behavioral plasticity to repeated cocaine administration. Neuron. 2014;82(3):645-58.

78 Berman RF, Buijsen RA, Usdin K, Pintado E, Kooy F, Pretto D, et al. Mouse models of the fragile $\mathrm{X}$ premutation and fragile $\mathrm{X}$-associated tremor/ataxia syndrome. J Neurodev Disord. 2014;6(1):25-16.

79 Irwin SA, Galvez R, Greenough WT. Dendritic spine structural anomalies in fragile- $\mathrm{X}$ mental retardation syndrome. Cereb Cortex. 2000;10(10):1038-44.

80 Coffee B, Zhang F, Ceman S, Warren ST, Reines D. Histone modifications depict an aberrantly heterochromatinized FMR1 gene in fragile x syndrome. Am J Hum Genet. 2002; 71(4):923-32.

81 Chiurazzi P, Pomponi MG, Willemsen R, Oostra BA, Neri G. In vitro reactivation of the FMR1 gene involved in fragile $\mathrm{X}$ syndrome. Hum Mol Genet. 1998;7(1):109-13.

82 Coffee B, Zhang F, Warren ST, Reines D. Acetylated histones are associated with FMR1 in normal but not fragile $\mathrm{X}$-syndrome cells. Nature Genet. 1999;22:98-101. Erratum in: Nat Genet. 1999;22(2):209.

83 Biacsi R, Kumari D, Usdin K. SIRT1 inhibition alleviates gene silencing in Fragile $\mathrm{X}$ mental retardation syndrome. PLoS Genet. 2008;4(3):e1000017-9.

84 Liu XS, Wu H, Krzisch M, Wu X, Graef J, Muffat J, et al. Rescue of Fragile X syndrome neurons by DNA methylation editing of the FMR1 gene. Cell. 2018;172(5):979-92.

85 Rauch BJ, Silvis MR, Hultquist JF, Waters CS, McGregor MJ, Krogan NJ, et al. Inhibition of CRISPR-Cas9 with Bacteriophage Proteins. Cell. 2017;168(1-2):150-e10.

86 Wu SC, Zhang Y. Active DNA demethylation: many roads lead to Rome. Nat Rev Mol Cell Biol. 2010;11(9):607-20.

$87 \mathrm{Wu} \mathrm{H}$, Zhang Y. Reversing DNA methylation: mechanisms, genomics, and biological functions. Cell. 2014;156(1-2):45-68.

88 Park CY, Halevy T, Lee DR, Sung JJ, Lee JS, Yanuka O, et al. Reversion of FMR1 methylation and silencing by editing the triplet repeats in Fragile X iPSC-derived neurons. Cell Rep. 2015;13(2):234-41.

89 Xie N, Gong H, Suhl JA, Chopra P, Wang T, Warren ST. Reactivation of FMR1 by CRISPR/Cas9-mediated deletion of the expanded CGG-repeat of the fragile X chromosome. PLoS One. 2016;11(10):e165499-12.

90 Conne B, Stutz A, Vassalli JD. The 3' untranslated region of messenger RNA: a molecular "hotspot" for pathology?. Nat Med. 2000;6(6): 637-41. 
91 Parker R, Sheth U. P bodies and the control of mRNA translation and degradation. Mol Cell. 2007;25(5):635-46.

92 Ramocki MB, Tavyev YJ, Peters SU. The MECP2 duplication syndrome. Am J Med Genet A. 2010;152A(5):1079-88.

93 Luoni $M$, Giannelli S, Indrigo M, Niro A, Massimino L, Iannielli A, et al. Whole brain delivery of an instability-prone Mecp2 transgene improves behavioral and molecular pathological defects in mouse models of Rett syndrome. eLife. 2020;9:e52629.
94 Hanlon KS, Kleinstiver BP, Garcia SP, Zaborowski MP, Volak A, Spirig SE, et al. High levels of AAV vector integration into CRISPR-induced DNA breaks. Nat Commun. 2019;10(1):4439-11.

95 Nelson CE, Wu Y, Gemberling MP, Oliver ML, Waller MA, Bohning JD, et al. Long-term evaluation of AAV-CRISPR genome editing for Duchenne muscular dystrophy. Nat Med. 2019;25(3):427-32.

96 Wolter JM, Mao H, Fragola G, Simon JM, Krantz JL, Bazick HO, et al. Cas9 gene therapy for Angelman syndrome traps Ube3a-ATS long non-coding RNA. Nature. 2020; 587(7833):281-4

97 Wang D, Mou H, Li S, Li Y, Hough S, Tran K, et al. Adenovirus-mediated somatic genome editing of Pten by CRISPR/Cas9 in mouse liver in spite of Cas9-specific immune responses. Hum Gene Ther. 2015;26(7):432-42.
98 Ran FA, Cong L, Yan WX, Scott DA, Gootenberg JS, Kriz AJ, et al. In vivo genome editing using Staphylococcus aureus Cas9. Nature. 2015;520(7546):186-91.

99 Fine EJ, Appleton CM, White DE, Brown MT, Deshmukh H, Kemp ML, et al. Transspliced Cas9 allows cleavage of $\mathrm{HBB}$ and CCR5 genes in human cells using compact expression cassettes. Sci Rep. 2015;5(May): 10777-9.

100 Chew WL, Tabebordbar M, Cheng JK, Mali $\mathrm{P}, \mathrm{Wu} \mathrm{EY}, \mathrm{Ng} \mathrm{AH}$, et al. A multifunctional AAV-CRISPR-Cas9 and its host response. Nat Methods. 2016;13(10):868-74. 